\title{
Optimizing the control system of a single-axis sun tracking mechanism
}

\author{
Cătălin Alexandru*
}

Transilvania University of Brașov, Product Design, Mechatronics and Environment Department, 500036 Brașov, Romania

\begin{abstract}
The work shows the optimization of the control system for the single-axis solar tracker that equips a solar panel, with the aim to increase the energetic efficiency of the system by maximizing the quantity of incident solar radiation that is captured - absorbed by the panel. The single-axis solar tracker is driven by a linear actuator, the optimization study intending to determine the optimal configuration (in terms of tuning factors) of the controller, which is a PID (Proportional- Integral-Derivative) device, in order to accurately achieve the motion (tracking) law imposed on the solar panel. The solar tracker was approached as a mechatronic system, the mechanical device (developed in ADAMS - Automatic Dynamic Analysis of Mechanical Systems) and the control system (developed in EASY5 - Engineering Analysis System) being integrated at the level of virtual prototype, in the concurrent engineering concept.
\end{abstract}

\section{Introduction}

Virtual prototyping is a modern modeling, simulation and optimization technique, which is based on conception of complex virtual models, close to the real ones [1-4]. This technique is being used successfully in domains established as being suitable for testing in virtual environment (e.g. automotive, aerospace, railways), but also in new interdisciplinary areas, such as the renewable energy systems (RES). Among the RES applications, the design and testing of the mechatronic tracking systems used for the solar panels/collectors is appropriate to be addressed through the use of specific virtual prototyping tools [5-11].

The main issue to be considered when designing solar tracking systems is the energetic and economic efficiency, and in this area virtual prototyping offers a great support. The optimization of the solar tracking systems can be focused in two directions, corresponding to the two main components of a mechatronic system: the mechanical device, and the actuating \& control system. The optimization of the mechanical device intends to minimize the geometric configuration of the tracking mechanism so that to minimize the motor forces/torques generated by the actuating sources to allow the panel to follow as accurately the trajectory of the sun on the celestial vault, thus minimizing the energy/power consumption for performing the tracking. On the other hand, the optimization of the control system intends to minimize the tracking errors (i.e. the differences between the imposed and current trajectories of the solar panel), thus maximizing the amount of incident solar radiation captured by the solar panel.

The present work is focused on the optimization of the control system for a single-axis solar tracker, whose dynamic virtual model was developed in mechatronic concept by using a virtual prototyping platform that integrates three types of software solutions CATIA - to create the 3D-solid model of the mechanism, ADAMS to design and simulate the multi-body system (MBS) model, EASY5 - to design the control system block diagram. The specific data have been integrated at the level of MBS model conceived in ADAMS, which was also used for the dynamic optimization of the solar tracking mechanism.

\section{Setting the optimization problem}

In ADAMS, there are the following steps for performing the optimization of a mechanical or mechatronic systems: parameterizing the virtual model; modeling the design variables, constraints and objectives; carrying out parametric design studies to identify the main design variables (with significant influence on the dynamic behavior of the tracking system, which is expressed by the design objectives); optimizing the virtual model by identifying the optimal values of the main design variables that assure, by case, the minimization or maximization of the design objective functions.

In the present work, the aim of the optimization consists in the minimization of the tracking errors, as stated in the first section of the paper. The case study is developed for a single-axis tracking mechanism, with one degree of freedom (DOF), corresponding to the daily motion of the solar panel, while the elevation is maintained at a fixed optimal position, corresponding to the local geographic area. The actuating source of the tracking mechanism is a linear actuator, whose disposing was the subject of optimization from the point of view of the mechanical device [12].

\footnotetext{
* Corresponding author: calex@unitbv.ro
} 


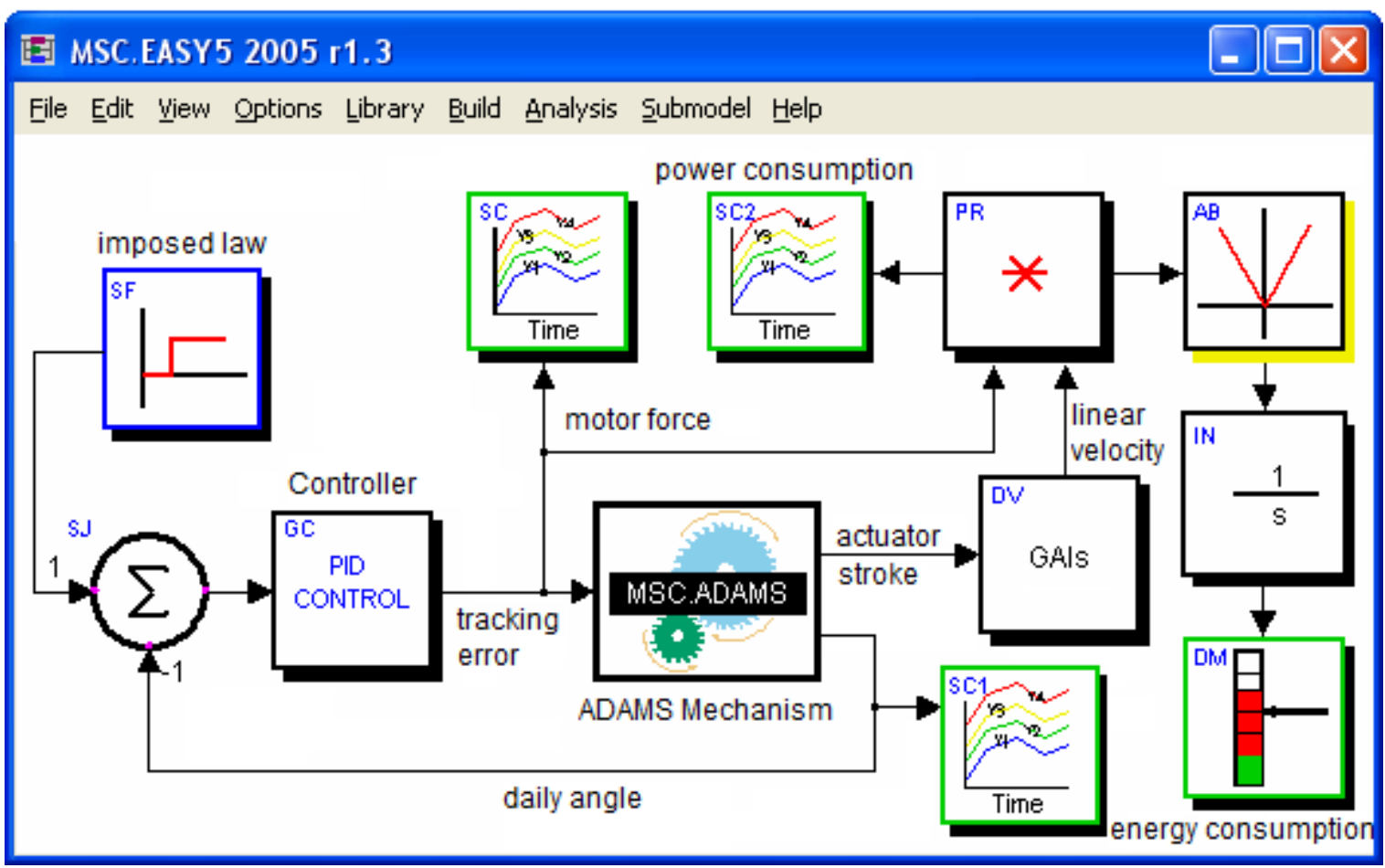

Fig. 1. The control system block diagram of the single-axis tracking mechanism (EASY5).

The control system block diagram of the single-axis tracking mechanism, which was developed by using the DFC software solution EASY5, is shown in figure 1. The control system includes the ADAMS block interface (ADAMS mechanism), which represents the link to the MBS model of the solar tracker (shown in figure 2), whose detailed description was presented in [12].

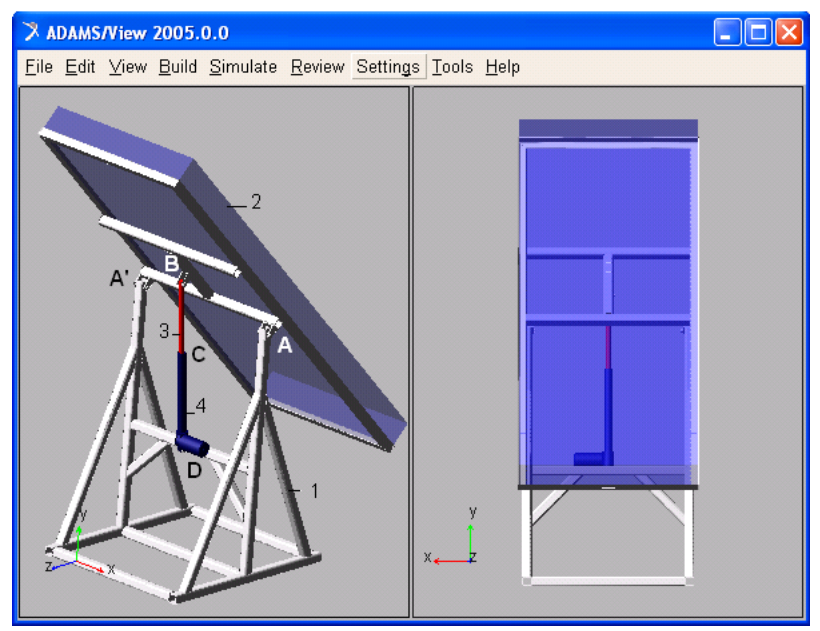

Fig. 2. The MBS model of the tracking mechanism (ADAMS).

The DFC block diagram shown in figure 1 is based on a single-loop control, the controlled parameter (which is input in the control system model) being represented by the daily angle of the solar panel, while the motor force developed by the linear actuator defines the input in the MBS (mechanical device) model. The daily angle is measured in the revolute joint A-A' between the solar panel and the fixed support, relative to the horizontal axis ( $\mathrm{X}$ in figure 2).
Excepting the ADAMS interface block, the following specific components (blocks) have been used in order to conceive the virtual dynamic model of the control system [13]:

- SF - step function generator, which generates a step signal that is used to model the imposed tracking law (i.e. the imposed daily angle of the solar panel);

- SJ - summing junction, which is used to compare the imposed daily angle (the output from the SF block) with the current/measured angle provided by the MBS model of the tracking mechanism, the result of the subtraction being the tracking error that will be minimized by the controller;

- GC - general controller, which models a PID (Proportional-Integral-Derivative) control element; the input in this block is the tracking error, while the output is represented by the motor force that will be applied as actuating element in the MBS mechanical model of the tracking mechanism;

- DV - derivative block, which is used to calculate the derivative of the input, in this case the actuator stroke (i.e. the relative displacement between the two components of the linear actuator - piston \& cylinder) that is provided by the MBS mechanical model, the output being the linear velocity in actuator;

- PR - product block, which calculates the product of two inputs, in this case the linear velocity in actuator and the motor force generated by the driving source, thus resulting the power consumption for performing the tracking (motion) law;

- AB - absolute block, which calculates the absolute value of the input parameter;

- IN - integrator block, which integrates the input signal with respect to time to produce the output signal, in

\footnotetext{
* Corresponding author: calex@unitbv.ro
} 
this case the energy consumption for performing the tracking;

- SC - strip chart, which is used to plot component outputs while the interactive simulation is running;

- DM - digital meter, which is used to digitally display the value of a component output.

It must be specified that for a complete step-by-step tracking law (formed by more tracking steps), the SF block can be replaced by a tabular function block ( $\mathrm{T} 1$ in EASY5), which allow to model the imposed variation of the daily angle as a function of time.

The PID controller is a control loop feedback device that assures important benefits, such as [14]: easy practical implementation, low cost, ability to solve various targets related to stationary error or overshoot, enable multi-loop control. Figure 3 shows the general scheme of the PID controller, the transfer function having the following form [13]:

$$
S_{-} O u t \_G C=E R I+G K P \cdot\left(R E F \_G C-F B S \cdot S \_F e e d b a c k\right)(1)
$$

where:

$$
\begin{gathered}
F B S=\frac{E R V+S \_ \text {Feedback } \cdot T C 1 \cdot G K F}{T C 2} \\
\frac{\mathrm{d}(E R V)}{\mathrm{d} t}=G K F \cdot S_{-} F e e d b a c k-F B S \\
\frac{\mathrm{d}(E R I)}{\mathrm{d} t}=G K I \cdot\left(R E F \_G C-F B S\right)
\end{gathered}
$$

with the following notations: REF - controller input (the tracking error); S Out - controller output (the motor force generated by the linear actuator, which is transmitted to the MBS mechanical model of the solar tracker); GKP proportional control gain; GKI - integration control gain; TC1 - derivative action time constant (which is used as a lag time constant to calculate an approximate derivative from the error signal); S_Feedback - controller feedback; TC2 - feedback damping time constant (which is used in the feedback line to help prevent an implicit loop); ERI integrated error signal; ERV - intermediate output; s Laplace transform.

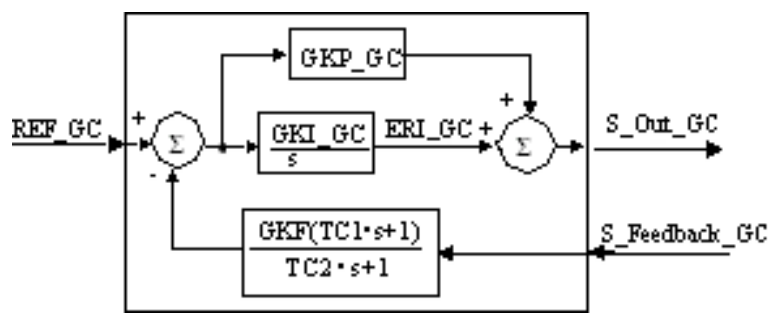

Fig. 3. The general model of the PID controller [13].

By neglecting the feedback line of the controller (which is integrated in the control system block diagram shown in figure 1), a simplified form of the transfer function of the PID controller is obtained, as follows:

$$
\frac{S \_O u t \_G C}{R E F \_G C}=G K P \cdot\left[1+\frac{1}{G K I \cdot s}+T C 1 \cdot s\right]
$$

The optimal design of the control system is defined by the following data: independent design variables - the tuning factors of the PID controller (GKP, GKI, TC1); design objective - the tracking error (the difference between the imposed and current/measured daily angles of the solar panel); monitored value of the design objective - the root mean square (RMS) during simulation; optimization purpose - the minimization of the design objective.

In the next stage, the control system model has been transfered from EASY5 to ADAMS, with the view to access the parametric optimization tools integrated in ADAMS (the same technique was used in [12] for the optimization of the mechanical device of the mono-axial tracking mechanism). In EASY5, the transfer is managed through the ADAMS External System Library (ESL) interface, the so generated ESL file being then imported in ADAMS as a general state equation [15]. In this way, the virtual prototype (including MBS \& DFC components) is ready for the dynamic optimization. Each design variable (GKP, GKI, TC1) is defined by an initial value, and a variation domain / range limits [minimum value, maximum value].

Thus, a mono-objective optimization problem is configured with the aim to seek the optimal values of the three tuning factors of the PID controller in order to achieve the objective pursued by minimizing the tracking error (in terms of root mean square during simulation), thus increasing the energetic efficiency of the tracking system (by maximizing the rate of incident radiation that is captured by the solar panel).

\section{Results and conclusions}

For this research, the numerical computations (simulations) have been carried out by considering the data specific to the Braşov geographical area (latitude 45.6333 North, longitude - 25.5833 East), on the day of the spring echinoctium (March, 21), the tracking law being configured in line with those presented in [9]. The angular domain for the daily motion is $\beta^{*} \in\left[60^{\circ},-60^{\circ}\right]$, where the daily angle $\beta^{*}$ is null when the panel reaches the solar noon position, with 8 equal motion steps of angular size $\Delta \beta^{*}=15^{\circ}$. In these terms, the time-history variation of the daily angle of the solar panel (i.e. the stepby-step tracking law) is shown in figure 4 .

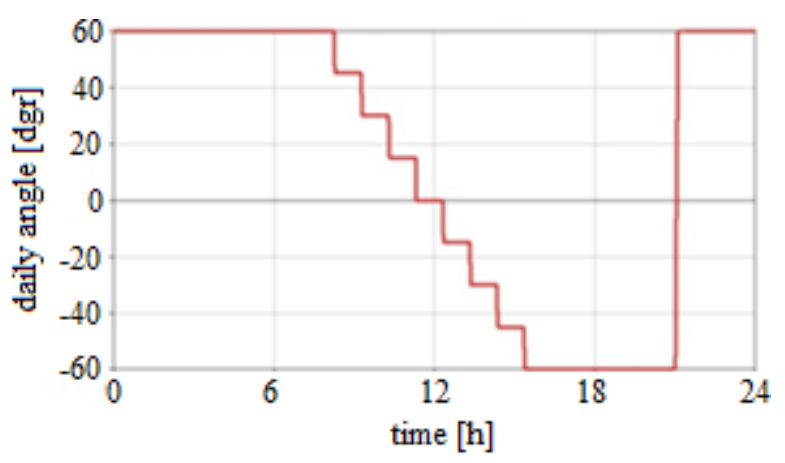

Fig. 4. The step-by-step tracking law. 
The OPTDES-GRG generalized reduced gradient algorithm, which is provided with ADAMS/View, was used to find the optimal design of the PID control element. The solution is obtained by combining the values of the design variables within the specific variation domains, the optimal design corresponding to the set of values that assure the minimal root mean square during simulation of the tracking errors (namely, GKP $=2200$, GKI $=1530$, $\mathrm{TC} 1=1500$ ), the time-history variation of the tracking error shown in figure 5 proving the performance of the so obtained optimal solar tracker. Thus, the optimization of the control system (controller) minimizes the tracking errors, with a positive effect on the energetic efficiency of the solar tracker, by maximizing the rate of incoming solar radiation.

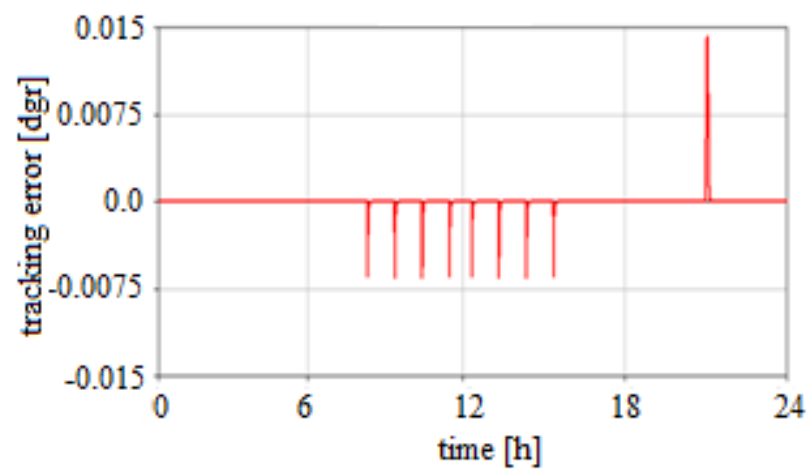

Fig. 5. The time-history variation of the tracking error.

As result of the dynamic simulation, two representative graphical simulation frames are shown in figure 6 , corresponding to the extreme positions of the system $\left(\beta^{*}= \pm 60^{\circ}\right)$, when the solar panel is oriented facing East (a), and respectively West (b).

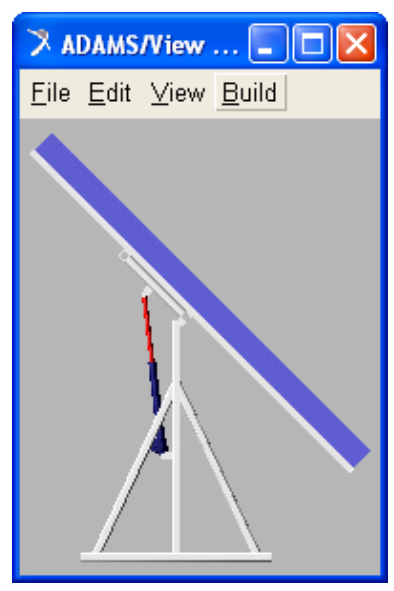

a.

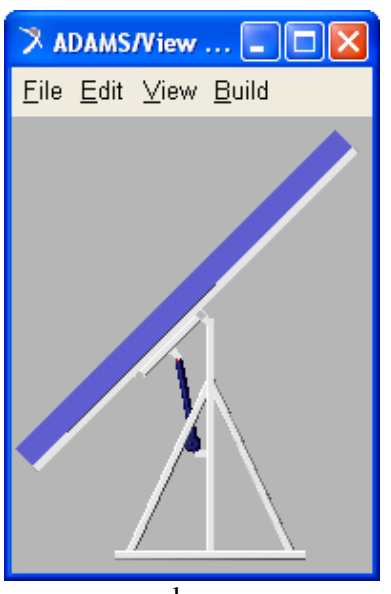

b.
Fig. 6. Graphical simulation frames.

Concluding, the study developed in this paper reveals the important benefits assured by the virtual prototyping in the solar trackers optimal design. The testing in virtual environment allows the comparative evaluation of a large variety of tracking strategies with the aim to improve the energy balance of the tracking mechanisms to much reduced costs compared to traditional experimental testing based on physical prototypes.

\section{References}

1. C. Alexandru, C. Pozna, C. Dynamic modeling and control of the windshield wiper mechanisms. WSEAS Transactions on Systems, 8, pp. 825-834 (2009)

2. C. Alexandru, P. Alexandru. The virtual prototype of a mechatronic suspension system with active force control. WSEAS Transactions on Systems, 9, pp. 927-936 (2010)

3. D. Tarniţă, D. Marghitu. Analysis of a hand arm system. Robotics and Computer-Integrated Manufacturing, 29, pp. 493-501 (2013)

4. D. Tarniţă, M. Catană, D.N. Tarniţă. Contributions on the modeling and simulation of the human knee joint with applications to the robotic structures. Mechanisms and Machine Science, 20, pp. 283-297 (2014)

5. C. Alexandru, M. Comşiţ. The energy balance of the photovoltaic tracking systems using virtual prototyping platform. 5th IEEE International Conference on the European Electricity Market EEM, pp. 253-258 (2008)

6. C. Alexandru. The design and optimization of a photovoltaic tracking mechanism. 2nd IEEE International Conference on Power Engineering, Energy and Electrical Drives POWERENG, pp. 436441 (2009)

7. D.A. Flores-Hernández, S. Palomino-Resendiz, N. Lozada-Castilloc, A. Luviano-Juárez. Mechatronic design and implementation of a two axes sun tracking photovoltaic system driven by a robotic sensor. Mechatronics, 47, pp. 148-159 (2017)

8. F.M. Hoffmann, R.F. Molz, J.V. Kothe, E.O.B. Nara, L. Pablo, C. Tedesco. Monthly profile analysis based on a two-axis solar tracker proposal for photovoltaic panels. Renewable Energy, 115, pp. $750-759$ (2018)

9. C. Alexandru, I.N. Tatu. Optimal design of the solar tracker used for a photovoltaic string. Journal of Renewable and Sustainable Energy, 5, pp. 1-16 (2013)

10. S. Seme, B. Stumberger, M. Hadziselimovic. A novel prediction algorithm for solar angles using second derivative of the energy for photovoltaic sun tracking purposes. Solar Energy, 137, pp. 201-211 (2016)

11. S.A. Sharaf Eldin, M.S. Abd-Elhady, H.A. Kandil. Feasibility of solar tracking systems for PV panels in hot and cold regions. Renewable Energy, 85, pp. 228233 (2016)

12. C. Alexandru. Optimizing the mechanical device of a mono-axial sun tracking mechanism. Submitted to IMT 2018

13. *** EASY5 library user guides. MSC Software, 2005

14. M.M. Sabir, T. Ali. Optimal PID controller design through swarm intelligence algorithms for sun tracking system. Applied Mathematics and Computation, 274, pp. 690-699(2016)

15. *** Getting started using ADAMS/Controls. MSC Software, 2005 